\title{
Adaptation to displaced vision contingent upon vibrating stimulation'
}

JEROME H. KRAVITZ AND HANS WALLACH

SWARTHMORE COLLEGE

In two experiments Ss were exposed to a prismatically displaced view of their right hand resting on a board that could be made to vibrate. Groups receiving vibration achieved significantly more adaptation as measured by a pointing test than control groups who did not.

A wedge prism placed in front of the eye produces a displacement of visual directions and corresponding errors in the localization of objects. Many investigators have reported adaptation in the form of errors in localizing responses after the prism is removed. The re-afference hypothesis, introduced by Held (Held \& Freedman, 1963), ascribes such adaptation to selfproduced movements which provide a re-afferent feedback. Also applicable is the cue-discrepancy hypothesis, proposed by Wallach \& Karsh (1963). Both hypotheses are concerned with the necessary condition of perceptual adaptation which takes place when some device is worn that causes the stimulation reaching a sense organ to misrepresent some property of the objective environment. Specifically, they provide an answer to the question: what kind of information enables the nervous system to achieve that adaptation, i.e., to learn to compensate for the misrepresentation of the object environment which results from the device. The re-afference hypothesis states that only the results of self-produced movement provide information useful for achieving adaptation. The cue-discrepancy hypothesis claims that any kind of stimulation that represents the critical property of the environment veridically, i.e., unaffected by the interfering device, may supply that information, even when $S$ remains immobile. That stimulation unaffected by the device may reach an inactive $S$ is due to the fact that, in humans, numerous perceptual properties are mediated by more than one condition of stimulation. Consequently, where normally two different conditions of stimulation give rise to the same perceptual property, one of them may be artificially altered to misrepresent the environment. If the other is permitted to operate, adaptation may take effect: one or both of the two perceptual processes that are caused by the two conditions of stimulation may be modified in such a way that their results come to be in better agreement with each other than initially when they fully reflected the cue discrepancy. If this modification takes place in that process which arises from the artificially altered stimulation, it amounts to adaptation.

There are instances of adaptation where only the cue-discrepancy hypothesis is applicable because adaptation can be shown to take place while $S$ remains pas- sive. But in the area of adaptation to displaced visual direction there is strong evidence that adaptation requires self-produced movements. In an experiment by Held \& Hein (1958), $\mathrm{S}$ is given a prismatically displaced view of his hand under three different exposure conditions: (1) with the arm at rest, (2) with the arm passive, but moved back and forth by an external agent, and (3) with the arm in active motion. As measured in a test in which $\mathrm{S}$ has to mark a number of visual targets while he is unable to see his operating hand, only the active movement condition produced an adaptation. This result is a challenge to the cue-discrepancy hypothesis, because the experiment is clearly within its province. Two sets of cues represent the location of the hand, proprioceptive and visual ones. With the visual cues misrepresenting the hand's location because of the prismatic displacement of its visual direction, a discrepancy between the visual and the proprioceptive cues exists which could lead to adaptation. It seems that Held and Hein's result that adaptation is obtained only with active arm movements is a clear confirmation of the re-afference principle. To avoid such a conclusion it would be necessary to argue that the active and passive conditions differ functionally in more than one way and that self-produced movement as such is not the decisive condition. Our experiments are an attempt to furnish support to such arguments. Procedure

The experiments compared the effectiveness of two types of "passive" training conditions in which $S$ was exposed to a prismatically displaced view of his stationary right forearm. Each experiment employed a total of $40 \mathrm{Ss}$ in four conditions of $10 \mathrm{Ss}$ each. In two conditions $(w / V)$ vibratory stimulation was applied during training to the S's prismatically viewed forearm, and in two $n / V$ conditions vibration was absent. In one $w / V$ and one $\mathrm{n} / \mathrm{V}$ condition the prismatic displacement was downward (D), while in the other two the displacement was upward (U). Two such experiments we re conducted; they differed only with respect to the position of the forearm relative S's eyes during training. In the first experiment Ss forearm was resting on a platform $98 \mathrm{~cm}$ above the floor. When it was found that this condition had produced asymmetrical results, the experiment was repeated with the forearm more nearly at normal table height, namely, $77 \mathrm{~cm}$ above the floor.

In the 10 min. training period, $S$ was seated, his right forearm resting on a platform which could be made to vibrate at 30 cycles per sec. $S$ was instructed to sit relaxed and to look at his right forearm; no biteboard 
was employed. A 30 diopter wedge prism covered with a Wratten filter 58 and attached to the right-eye opening of welder's goggles was worn during the training period. S's left eye was occluded throughout.

To measure the effects of training two different test were employed, a pointing test (Ptg) and a horizontal direction (HD) test. Each test was given twice during the course of the experiment, once before and again after training. In all cases they were made in the dark and without the prism. The difference between the pre- and post-training test measured the adaptation. In both tests $S$ stood with his head position fixed by a biteboard. In the Ptg test he was asked to point with extended right arm at a luminous target, a horizontal line 10 by $0.5 \mathrm{~cm}$, which was located approximately at eye level at a distance of $1 \mathrm{~m}$ straight in front of him. In the HD test, $E$ moved the target line slowly up or downward until $\mathrm{S}$ judged it to be straight ahead, that is, at the level of his eyes. Each HD test consisted of two such settings, one for which the starting position of the line was above and the other in which $\mathrm{E}$ gradually raised the line from below toward the horizontal position, and the test result was the mean of the two settings. E moved the target line in conformity with $S$ 's instructions. Since this took place in the dark, $E$ was unaware of the exact position of the target line, until a setting was completed and he read the position with a flashlight. The order of the initial target position was counterbalanced. The test order was Ptg followed by HD before training and HD followed by Ptg after training.

\section{Results}

The adaptation effects obtained from each group of 10 Ss are given in Table I. Each number is the mean of the individual differences between the pre- and posttraining test results. Where this difference was in the direction opposite to adaptation it was represented by a negative number. The HD tests showed no adaptation: mean effects in the direction of adaptation were obtained only where the prismatic displacement was downward. In the condition that yielded the best adaptation effect on the Ptg test (normal arm level, w/V), the mean HD effects for the upward and the downward displacing prism canceled each other out. We have obtained similar results for HD tests in other experiments with adaptation to vertically displacing prisms and we will discuss their effects in a subsequent publication.

Table 1. Mean adaptation in degrees: average of differences between test results obtained before and after adaptation.

\begin{tabular}{|c|c|c|c|c|c|c|c|c|}
\hline \multirow{2}{*}{ Condition } & \multicolumn{4}{|c|}{ High Arm Level } & \multicolumn{4}{|c|}{ Normal Arm Level } \\
\hline & $\mathrm{Ptg}$ & $p$ & HD & $\mathrm{p}$ & Ptg & $\mathrm{p}$ & HD & $p$ \\
\hline \multicolumn{9}{|c|}{ No Vibration } \\
\hline $\mathrm{U}_{p}$ & 1.79 & .2 & -0.08 & & 0.68 & .4 & -0.91 & .4 \\
\hline Down & 1.90 & .3 & 3.39 & .05 & 2.20 & .1 & 1.80 & .1 \\
\hline \multicolumn{9}{|c|}{ With Vibration } \\
\hline $\begin{array}{l}\text { Up } \\
\text { Down }\end{array}$ & $\begin{array}{l}1.75 \\
6.80\end{array}$ & $\begin{array}{l}.05 \\
.001\end{array}$ & $\begin{array}{l}0.10 \\
5.01\end{array}$ & .001 & $\begin{array}{l}3.15 \\
3.54\end{array}$ & $\begin{array}{l}.01 \\
.001\end{array}$ & $\begin{array}{r}-2.21 \\
1.62\end{array}$ & $\begin{array}{l}.06 \\
.05\end{array}$ \\
\hline
\end{tabular}

With the pointing test, significant adaptation was measured for all four groups whose training was with vibration, although under the high arm condition the effect of the downward displacement was significantly larger $(p<.02)$ than the effect of the upward displacing prism. Training with the arm at nearly normal table level eliminated this asymmetry. In the case of the latter experiment the combined adaptation effect for the upward and downward displacement conditions was significantly different for the $\mathrm{n} / \mathrm{V}$ and $\mathrm{w} / \mathrm{V}$ conditions. With vibration applied during training the combined effects were $1.9^{\circ}(p<.05)$ higher than the corresponding mean for the two groups who received novibration. A similar difference for the combined results of both experiments $\left(2.1^{\circ}\right)$ was significant at the .01 level of confidence. Discussion

Significant adaptation effects were obtained when Ss observed their immobile forearm through a displacing prism while the arm was resting on a vibrating board. This requires a different interpretation of Held and Hein's experiment: self-produced movements of an arm are not the only condition under which adaptation to prismatic displacement can be obtained by looking at one's forearm. We believe that both, self-produced movements and vibrating stimulation, operate by enhancing the central effect of proprioceptive stimulation representing the true arm position.

In the case of the high arm position the effect of adaptation to prismatic displacement is overlayed by a partial adaptation to the abnormal arm position. The abnormally high position which the armoccupies during the training period causes pointing in the post-training test to be too high. The latter fact becomes evident when the adaptation effects $w / V$ for the two arm positions are compared with the adaptation direction in mind. Inasmuch as adaptation to the U-prism is measured as a change in the pointing direction downward and adaptation to a D-prism as a pointing change upward, the diminished effect of adaptation to the Uprism $\left(1.75^{\circ}\right.$ for the high arm condition vs. $3.15^{\circ}$ for normal arm position) and the enhanced effect obtained with the D-prism $\left(6.80^{\circ}\right.$ vs. $\left.3.54^{\circ}\right)$ amount to a tendency to point too high after training under the high arm condition. It is interesting that the adaptation to the high arm position also is brought out by vibration $(p<.06)$.

\section{References}

Held, R., \& Freedman, S. J. Plasticity in human sensorimotor control. Science, 1963, 142, 455-462.

Held, R., \& Hein, A. Adaptation of disarranged hand-eye coordination contingent upon re-afferent stimulation. Percept. mot. Skills, $1958,8,87-90$.

Wallach, H., \& Karsh, E. B. The modification of stereoscopic depth-perception and the kinetic depth-effect. Amer. J. Psychol., $1963,76,429-435$

\section{Note}

1. This work was supported by a grant from the National Science Foundation to Swarthmore College, Hans Wallach principal investigator. 